\title{
On the Interpretation of Ground Reflections Observed in Small-Scale Experiments Simulating Lightning Strikes to Towers
}

\author{
Yoshihiro Baba, Member, IEEE, and Vladimir A. Rakov, Fellow, IEEE
}

\begin{abstract}
Using the finite-difference time-domain (FDTD) method for solving Maxwell's equations, we have simulated smallscale experiments intended to study the interaction of lightning with towers. In these experiments, employing the time-domain reflectometry (TDR), the tower was represented by a conical conductor placed between two horizontal conducting planes, and a relatively high grounding impedance (about $60 \Omega$, constant or decreasing with time) of the bottom plane was inferred, based on the assumption that a conical conductor could support propagation of unattenuated waves in either direction. We have shown, using the FDTD simulations, that a current pulse suffers no attenuation when it propagates downward from the apex of the conical conductor to its base, but it attenuates significantly when it propagates upward from the base of the conical conductor to its apex. We show that the current reflection coefficient at the base of the conical conductor is close to 1 , so that the equivalent grounding impedance of the conducting plane is close to zero. Our analysis suggests that the relatively high grounding impedance of conducting plane inferred from the small-scale experiments is an engineering approximation to the neglected attenuation of upward propagating waves. When the dependence of cone's waveguiding properties on the direction of propagation is taken into account, the results of small-scale experiments simulating lightning strikes to towers can be interpreted without invoking the fictitious grounding impedance of conducting plane. Representation of a vertical strike object by a uniform transmission line terminated in a fictitious grounding impedance appears to be justified in computing lightning-generated magnetic fields and relatively distant electric fields, but may be inadequate for calculating electric fields in the immediate vicinity of the object. This study was motivated by the growing interest in extending lightning return stroke models to include a tall strike object and calculating associated electric and magnetic fields.
\end{abstract}

Index Terms-Biconical antenna, finite-difference time-domain (FDTD) method, ground reflection, lightning current, tall objects.

\section{INTRODUCTION}

D IRECT measurements of lightning currents on grounded tall objects (e.g., Berger et al. [1], Gorin et al. [2], Montandon and Beyeler [3], Zundl [4], Janischewskyj et al. [5], Goshima et al. [6]) have provided important data on lightning current parameters and the equivalent impedance of lightning channel. Lightning current waveforms measured on a grounded tall object may be influenced by reflections at the bottom and

Manuscript received April 22, 2004; revised January 13, 2005. This work was supported in part by Doshisha University and by National Science Foundation under Grants ATM-0003994 and ATM-0346164.

Y. Baba is with the Department of Electrical Engineering, Doshisha University, Kyoto 610-0321, Japan (e-mail: ybaba@mail.doshisha.ac.jp).

V. A. Rakov is with the Department of Electrical and Computer Engineering, University of Florida, Gainesville, FL 32611 USA (e-mail: rakov@ece.ufl.edu).

Digital Object Identifier 10.1109/TEMC.2005.852220 top of the object. If a grounded tall object struck by lightning can be represented by a transmission line and current reflection coefficients at the top and bottom of the object are known, it is possible to evaluate lightning current that is not influenced by the presence of the object from measured lightning current (e.g., Rakov [7], Rachidi et al. [8], Bermudez et al. [9]). Validity of the transmission-line representation of a grounded tall object struck by lightning is investigated by comparing the modelpredicted field waveforms (lightning current measured at the top of the object is usually used as an input) with the measured field waveforms (e.g., Zundl [4], Motoyama et al. [10], Rakov and Uman [11], Goshima et al. [6]). A uniform, lossless transmission line representation is usually used, although a vertical conductor above ground generally cannot be viewed as a uniform transmission line (e.g., Jordan [12], corrected by Wagner and Hileman [13], Baba and Rakov [14]). Also, in analyzing lightning surges on overhead power transmission lines struck by lightning, representation of a transmission-line tower by a short uniform lossless transmission line terminated at its bottom end in the tower grounding impedance (e.g., Sargent and Darveniza [15], Chisholm et al. [16]) has been widely used. Several formulas for the characteristic impedance of grounded strike object (tower) are found in the literature. In simplified analyses (e.g., Sargent and Darveniza [15], Chisholm et al. [16], Bermudez et al. [17]), the characteristic impedance derived for an ideal biconical antenna (e.g., Balanis [18]) has been used, apparently assuming that the lightning channel can be viewed as the upper part of such an antenna and the strike object as its lower part.

An ideal biconical antenna (or waveguide) comprises two concentric conical conducting surfaces of infinite extent with common apexes. It has constant input impedance equal to its characteristic impedance (e.g., Balanis [18]). Thus, if it is excited by a voltage or current source connected at its input terminals (between the cone apexes), two current pulses propagate, without attenuation or dispersion, away from the apexes along the surfaces of the cones. The electromagnetic field structure associated with these unattenuated current pulses is spherical TEM. It follows from the image theory that a vertical inverted conical conductor of infinite extent above a horizontal conducting plane or a vertical conical conductor of infinite extent below a horizontal conducting plane, excited at its apex, is equivalent to an ideal biconical antenna (except for the value of input impedance; Balanis [18]). If the conical conductor (or inverted conical conductor) is not infinitely long (it may be truncated or be connected to a conductor of different geometry, for 
example, conducting plane), a reflected current pulse is generated at the cone termination and propagates toward the cone apex. A biconical waveguide is not expected to behave as a uniform transmission line for a reflected current pulse propagating toward the cone apex. This pulse should suffer attenuation, and the associated electromagnetic field structure should be nonTEM. To the best of our knowledge, the response of a biconical waveguide to a current pulse propagating toward the excitation point at its apex(es) has not been fully investigated yet. At least the dependence of waveguiding properties of a biconical antenna on the direction of wave propagation is generally not recognized in the lightning literature.

In this paper, using the finite-difference time-domain (FDTD) method (Yee [19], Taflove and Hagness [20]) for solving Maxwell's equations, we show that a current pulse suffers significant attenuation and dispersion when it propagates along a conical conductor toward its apex, in contrast with unattenuated and undispersed propagation away from the cone apex. We apply this fact to the interpretation of ground reflections observed in small-scale experiments simulating lightning strikes to towers. Note that a vertical conductor having a uniform thickness (such as a cylinder or parallelepiped) above ground is outside the scope of this paper. The propagation of current pulses along such a vertical conductor has recently been examined by Kordi et al. [21] and by Baba and Rakov [14].

The structure of the paper is as follows. In Section II, using the FDTD method, we demonstrate that a current pulse suffers no attenuation when it propagates along the surface of a conical conductor away from its apex, but it attenuates markedly when it propagates from the base of the conical conductor to its apex. In Section III, we compare the results of FDTD simulations with those of two small-scale experiments using a conical conductor placed between two horizontal conducting planes and excited at its apex, which were carried out by Chisholm and Janischewskyj [22] and by Bermudez et al. [17]. Both groups employed the time-domain reflectometry (TDR) to detect reflections from the bottom plane at the cone apex. In Section IV, we show that the relatively high grounding impedance of the bottom conducting plane inferred from the TDR measurements is fictitious and should be viewed as an engineering approximation to the neglected attenuation of current pulse as it propagates from the base of a conical conductor to its apex. Finally, in Section V, we examine the representation of a tall object on a conducting ground plane by a lossless uniform transmission line terminated in a fictitious, relatively high grounding impedance, as suggested by Bermudez et al. [17].

\section{Current Pulses Propagating Along a ConicAl CONDUCTOR EXCITED AT ITS APEX OR BASE}

Fig. 1(a) shows a vertical, perfectly conducting cone of base radius $8 \mathrm{~cm}$ placed between two horizontal perfectly conducting planes $40 \mathrm{~cm}$ apart, to be analyzed using the FDTD method. A current source, having a height of $1 \mathrm{~cm}$ and a cross-sectional area of $1.5 \times 1.5 \mathrm{~cm}^{2}$, is inserted between the cone apex and the top perfectly conducting plane. The source produces a Gaussian pulse having an amplitude of $1 \mathrm{~A}$ and a half-peak

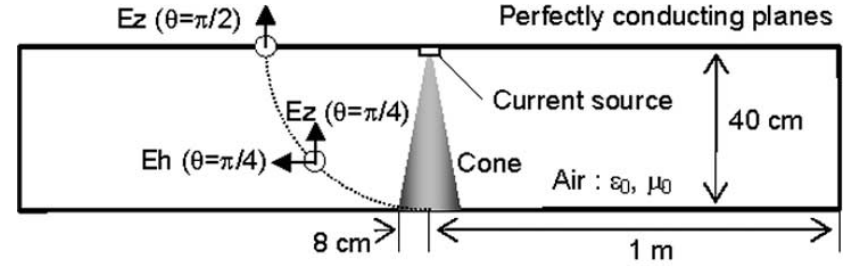

(a)

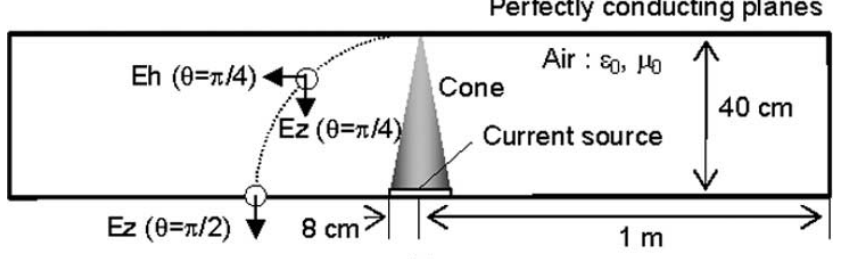

(b)

Fig. 1. (a) A perfectly conducting cone in air excited at its apex by a current source having a height of $1 \mathrm{~cm}$ and a cross-sectional area of $1.5 \times 1.5 \mathrm{~cm}^{2}$ and (b) that excited at its base by a current source having a height of $1 \mathrm{~cm}$ and an approximately circular cross-sectional area with a radius of $8.5 \mathrm{~cm}$, to be analyzed using the FDTD method. The current source produces a Gaussian pulse having an amplitude of $1 \mathrm{~A}$ and a half-peak width of $0.33 \mathrm{~ns}$. The working volume of $2 \times 2 \times 0.4 \mathrm{~m}^{3}$, which is divided into $0.5 \times 0.5 \times 1 \mathrm{~cm}^{3}$ cells, is surrounded by six perfectly conducting planes.

width of $0.33 \mathrm{~ns}$. This current pulse propagates downward along the surface of the cone, away from its apex, until it encounters the bottom plane. Fig. 1(b) shows the same conical conductor but excited at its base by a current source having a height of $1 \mathrm{~cm}$ and an approximately circular cross-sectional area whose radius is $8.5 \mathrm{~cm}$. In this latter case, a current pulse propagates from the base of the conical conductor to its apex. The current source in the FDTD simulation is implemented by imposing the magnetic field vectors along the closest possible loop enclosing the current source (e.g., Baba and Rakov [14]). Currents and fields are calculated up to $2.5 \mathrm{~ns}$ with a time increment of $0.01 \mathrm{~ns}$. The working volume of $2 \times 2 \times 0.4 \mathrm{~m}^{3}$, shown in Fig. 1, is divided into $0.5 \times 0.5 \times 1 \mathrm{~cm}^{3}$ cells. Due to such rectangular discretization, the conical conductor in Fig. 1 has a staircase surface (see Fig. 15(a) in Appendix). The lateral dimensions of the volume are limited by perfectly conducting planes, which do not influence current waves propagating on the conical conductor for about 6 ns after current injection at its apex or base. This configuration is similar to that used in the small-scale experiments carried out by Chisholm et al. [16] and by Chisholm and Janischewskyj [22].

Fig. 2(a) shows current waveforms at different vertical distances from the apex of the conical conductor excited at the apex by a current source [see Fig. 1(a)], calculated using the FDTD method. Fig. 2(b) is similar to Fig. 2(a), but for the cone excited at its base. It is clear from Fig. 2(a) and (b) that the current pulse does not attenuate when it propagates from the cone apex to the cone base, but it attenuates markedly when it propagates from the cone base to the cone apex. Fig. 3(a) shows waveforms of vertical and horizontal electric fields at two points $40 \mathrm{~cm}$ away from the apex of the cone shown in Fig. 1(a), calculated using the FDTD method. Fig. 3(b) shows those at two points $40 \mathrm{~cm}$ away from the base center of the cone shown in Fig. 1(b). 


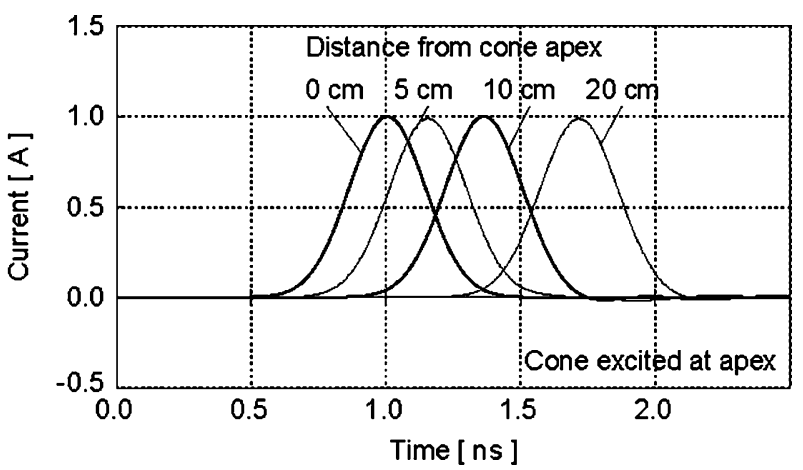

(a)

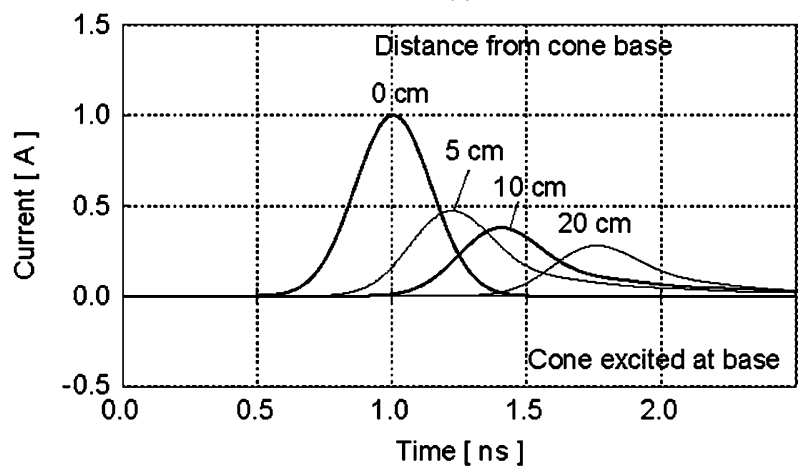

(b)

Fig. 2. Current waveforms for the conical conductor excited at its (a) apex and (b) base at different vertical distances from the current source, calculated using the FDTD method. Note that the current pulse propagates from the apex to the base (without attenuation) in (a) and from the base to the apex (with attenuation) in (b).

The electromagnetic field structure around an ideal biconical antenna, excited by a source connected between the cone apexes, is spherical TEM (e.g., Balanis [18]). The theta-directed electric field $E_{\theta}$ of the spherical TEM wave produced by an unattenuated current pulse $I$ propagating away from the excitation point of the biconical antenna is given by

$$
E_{\theta}(r, \theta, t)=\frac{1}{2 \pi \varepsilon_{0} \sin \theta} I(0, t-r / c)
$$

where $\varepsilon_{0}$ is the permittivity of vacuum, $c$ is the velocity of light, $r$ is the radial distance from the excitation point to the observation point, $\theta$ is the angle between the antenna axis and a straight line passing through both the excitation point and the observation point $(\theta$ is to be larger than or equal to the half-cone angle), and $I(0, t)$ is the source current. Equation (1) applies to the configuration presented in Fig. 1(a) until the current pulse arrives at the cone base. Equation (1) with $\theta \neq 0$ also applies to a zero-angle inverted cone above a conducting plane, that is, to an infinitely thin wire above aground (Thottappillil et al. [23]).

For the configuration shown in Fig. 1(a), the vertical and horizontal components, $E_{z}$ and $E_{h}$, of the electric field can be evaluated by multiplying (1) by $\cos (\pi / 2-\theta)$ and by $\sin (\pi / 2-\theta)$, respectively. For a spherical TEM wave, $E_{z}(r=40 \mathrm{~cm}, \theta=$ $\pi / 2), E_{z}(r=40 \mathrm{~cm}, \theta=\pi / 4)$, and $E_{h}(r=40 \mathrm{~cm}, \theta=\pi / 4)$ should be the same. For a source current pulse having a

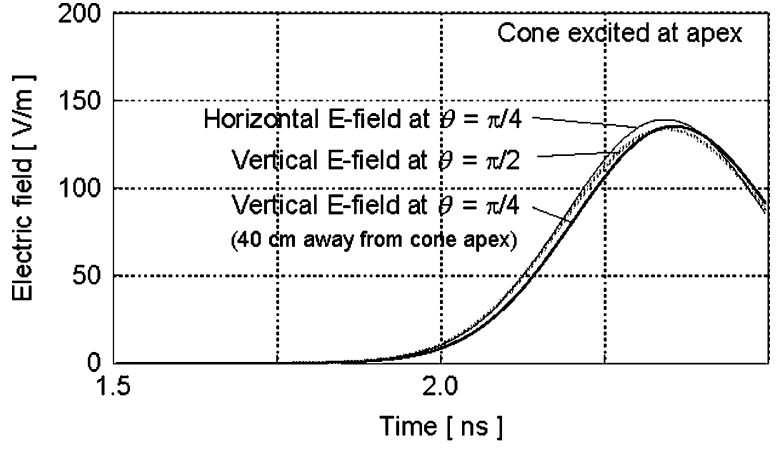

(a)

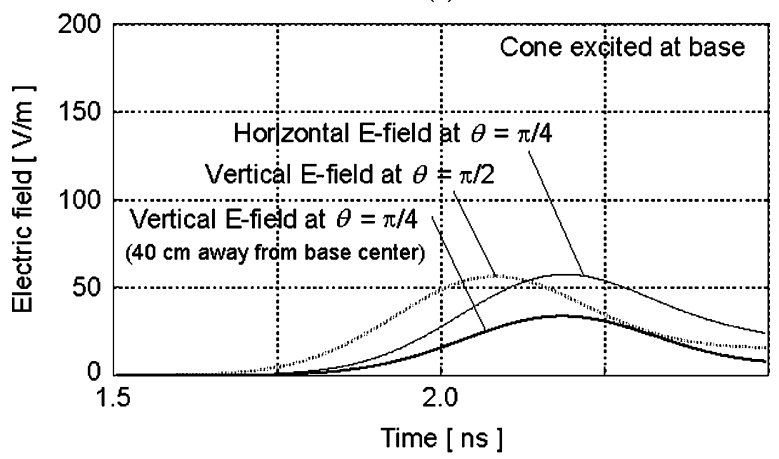

(b)

Fig. 3. Waveforms of vertical and horizontal electric fields at two observation points located (a) $40 \mathrm{~cm}$ away from the apex of the cone shown in Fig. 1(a) and (b) $40 \mathrm{~cm}$ away from the base center of the cone shown in Fig. 1(b). The source is located at the apex for (a) and at the base for (b). The fields are calculated using the FDTD method.

peak of $1 \mathrm{~A}$, the magnitude of these electric fields should be $150 \mathrm{~V} / \mathrm{m}$. This theoretical prediction for the configuration shown in Fig. 1(a) is to be compared with the corresponding electric field waveforms, calculated using the FDTD method and shown in Fig. 3(a). All three waveforms in Fig. 3(a) are very similar, which is consistent with the theoretical prediction, and magnitudes of these electric fields are only $7 \%$ to $10 \%$ less than the theoretical value $(150 \mathrm{~V} / \mathrm{m})$. Therefore, the electromagnetic field structure around the conical conductor excited at its apex is essentially spherical TEM until a reflection from the bottom perfectly conducting plane arrives at the observation point.

On the other hand, as seen in Fig. 3(b), electric field waveforms at the same observation points, calculated for the configuration shown in Fig. 1(b), differ considerably from each other. This indicates that the electromagnetic field structure around a conical conductor excited at its base is non-TEM. This implies that in the configuration shown in Fig. 1(a) a current wave reflected from the bottom plane also produces a non-TEM electromagnetic field structure.

In the following section, we will use the FDTD method to simulate two small-scale experiments conducted by Chisholm and Janischewskyj [22] and by Bermudez et al. [17] who used a conical conductor between two horizontal conducting planes to study the interaction of lightning with towers. These researchers have detected a lower than expected current at the apex of the conical conductor, via their voltage measurements at the top of 


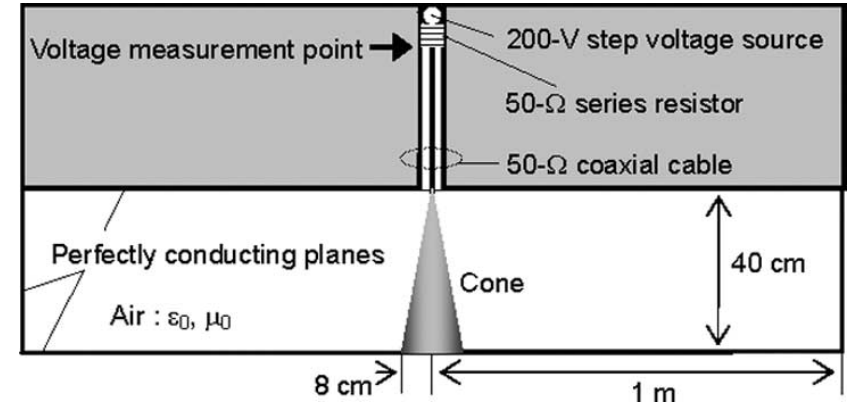

Fig. 4. Configuration used in FDTD analysis simulating an experiment carried out by Chisholm and Janischewskyj [22]. A perfectly conducting cone of a height of $40 \mathrm{~cm}$ and base radius of $8 \mathrm{~cm}$ is located between two horizontal conducting planes (see Fig. 15(a) in Appendix). The center conductor of a 50- $\Omega$ coaxial cable connects the apex of the conical conductor with a $200-\mathrm{V}$ step voltage source via a series $50-\Omega$ matching resistor. The outer shield of the $50-\Omega$ coaxial cable is connected to the top-conducting plane. The round-trip time of a wave propagating from the voltage measurement point to the lower end of the cable and back is $3.3 \mathrm{~ns}$. The working volume of $2 \times 2 \times 0.86 \mathrm{~m}^{2}$ is divided into $0.5 \times 0.5 \times 1 \mathrm{~cm}^{3}$ rectangular cells

the cone, and interpreted this result as being due to imperfect reflection from the bottom plane.

\section{FDTD SimULATION OF SMALL-SCALE EXPERIMENTS}

\section{A. 40-cm High-Conical Conductor (Chisholm and}

\section{Janischewskyj [22])}

Fig. 4 shows a perfectly conducting cone placed between two horizontal conducting planes $40 \mathrm{~cm}$ apart, to be analyzed using the FDTD method. This configuration simulates a small-scale TDR experiment carried out by Chisholm and Janischewskyj [22]. A step voltage of $200 \mathrm{~V}$ generated by the source (see Fig. 4) divides equally between the $50-\Omega$ series resistor and the $50-\Omega$ characteristic impedance of the coaxial cable connecting the source to the apex of the cone. As a result, a step voltage of $100 \mathrm{~V}(=200 \mathrm{~V} \times 50 \Omega /(50 \Omega+50 \Omega))$ is applied to the apex of the conical conductor. Voltage between the center conductor and the outer shield of this coaxial cable is monitored near the source (44 $\mathrm{cm}$ above the cone apex). The coaxial cable in this FDTD simulation has a square cross section. The side of the square center conductor is $1 \mathrm{~cm}$, and the inner side of the shield is $3 \mathrm{~cm}$. There is no formula for the characteristic impedance of such a cable. Hence, we evaluated its characteristic impedance from the ratio of the applied voltage and current, both calculated using the FDTD method. The characteristic impedance was estimated to be $55 \Omega$ when the relative permittivity of the medium between the center conductor and the shield was 1 . We set the relative permittivity of the medium to 1.21 in order to obtain $50-\Omega(=55 \Omega / \sqrt{ } 1.21)$ characteristic impedance for the simulated cable, which is equal to the characteristic impedance of the cable used in the experiment. The propagation velocity of a wave on this cable is $27 \mathrm{~cm} / \mathrm{ns}(=30 \mathrm{~cm} / \mathrm{ns} / \sqrt{ } 1.21)$. The round-trip time of a wave propagating from the voltage measurement point to the lower end of the cable (apex of the cone) and back is $3.3 \mathrm{~ns}(=2 \times 44 \mathrm{~cm} / 27 \mathrm{~cm} / \mathrm{ns})$, which is equal to that in the experiment. In order to reproduce the voltage waveform recorded at the source by Chisholm and Janischewskyj [22] and

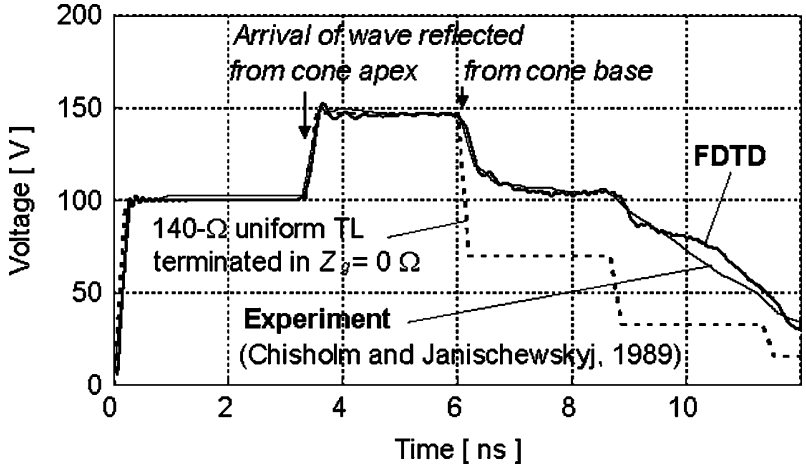

Fig. 5. Voltage waveforms at the top end of the 50- $\Omega$ cable (at the voltage source) measured (thinner solid line) by Chisholm and Janischewskyj [22] and calculated (thicker solid line) using the FDTD method for the configuration shown in Fig. 4. Also shown is the waveform (broken line) corresponding to the case of a $140-\Omega$ uniform transmission line short-circuited at its bottom end, calculated by Chisholm and Janischewskyj [22].

shown in Fig. 5 (thinner solid line), we performed calculations up to $12 \mathrm{~ns}$ with a time increment of $0.01 \mathrm{~ns}$.

Also shown in Fig. 5 (thicker solid line) is the voltage waveform calculated using the FDTD method for the configuration shown in Fig. 4. The calculated waveform agrees fairly well with the measured waveform. The first reflection from the junction between the $50-\Omega$ coaxial cable and the conical conductor arrives at the voltage measurement point around $3.3 \mathrm{~ns}$, and the second reflection from the bottom-conducting plane arrives there around $6 \mathrm{~ns}$. Magnitudes of measured and calculated voltages in Fig. 5 are almost constant from 0.2 to 3.3 ns because of the constant characteristic impedance of the cable. The constant magnitude of voltage from 3.4 to 6 ns indicates that the characteristic impedance of the cone is constant until the wave propagating downward from the cone apex encounters the bottom conducting plane. During this latter interval, the magnitude of the incident voltage calculated using the FDTD method is $V_{\text {inc }}=100 \mathrm{~V}$, and that of the reflected voltage is $V_{\text {refl }}=46 \mathrm{~V}$ $\left(=146 \mathrm{~V}-100 \mathrm{~V}\right.$; see Fig. 5). The relation between $V_{\text {refl }}$ and $V_{\text {inc }}$ is given by

$$
V_{\text {refl }}=\rho_{t} V_{\text {inc }}=\frac{Z_{\text {cone }}-Z_{\text {cable }}}{Z_{\text {cone }}+Z_{\text {cable }}} V_{\text {inc }}
$$

where $\rho_{t}$ is the voltage reflection coefficient at the top of the cone for downward propagating waves, $Z_{\text {cable }}(=50 \Omega)$ is the characteristic impedance of the cable, and $Z_{\text {cone }}$ (unknown quantity) is the characteristic impedance of the conical conductor below the horizontal conducting plane. From (2) with $V_{\text {inc }}=100 \mathrm{~V}$, $V_{\text {refl }}=46 \mathrm{~V}$, and $Z_{\text {cable }}=50 \Omega$, one can evaluate $Z_{\text {cone }}$ to be $135 \Omega$. The characteristic impedance of a conical conductor below a horizontal conducting plane excited at their junction is equal to one-half of the characteristic impedance of biconical antenna and given (e.g., Balanis 1997 [18]) by

$$
Z_{C}(\alpha)=60 \ln \left(\cot \frac{\alpha}{2}\right)
$$

where $\alpha$ is the half-cone angle. Since $\alpha=11.3^{\circ}\left(=\tan ^{-1}\right.$ $(8 / 40))$ for the cone shown in Fig. 4, its characteristic impedance, according to (3), is equal to $140 \Omega$. A similar 


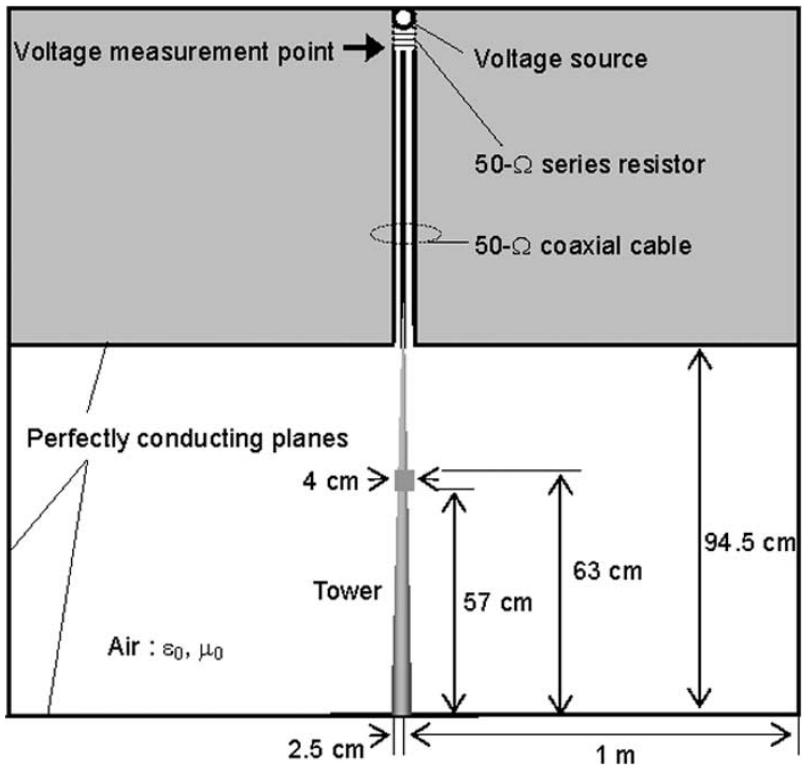

Fig. 6. Configuration used in FDTD analysis simulating an experiment carried out by Bermudez et al. [17]. A perfectly conducting cone of a height of $94.5 \mathrm{~cm}$ and base radius of $2.5 \mathrm{~cm}$, having a short bulge at a height of $60 \mathrm{~cm}$, is located between two horizontal conducting planes (see Fig. 15(b) in Appendix). The center conductor of a 50- $\Omega$ coaxial cable connects the apex of the cone with a voltage source via a series $50-\Omega$ matching resistor. The outer shield of the $50-\Omega$ coaxial cable is connected to the top conducting plane. The round-trip time of a wave propagating along the cable from the voltage measurement point to the lower end of the cable and back is $6 \mathrm{~ns}(=2 \times 81 \mathrm{~cm} / 27 \mathrm{~cm} / \mathrm{ns})$. The working volume of $2 \times 2 \times 1.785 \mathrm{~m}^{2}$ is divided into $0.5 \times 0.5 \times 1.5 \mathrm{~cm}^{3}$ rectangular cells.

value was obtained by Chisholm and Janischewskyj (1989 [22]) from their TDR measurements. The value of the characteristic impedance of the cone below the horizontal conducting plane obtained from the FDTD simulation, $135 \Omega$, is only $3 \%-4 \%$ less than the theoretical value given by (3) or the experimentally determined value.

Chisholm and Janischewskyj [22] have modeled the configuration of their small-scale experiment (see Fig. 4) by a 50$\Omega$ lossless uniform transmission line (representing the cable) connected in series with a $140-\Omega$ lossless uniform transmission line (representing the conical conductor) terminated in zero impedance (expected for a conducting plane). Using this model, they obtained a voltage waveform (broken-line curve in Fig. 5 ) at the source that coincided with the measured waveform shown in Fig. 5 for times up to 6 ns but dropped abruptly to about $70 \mathrm{~V}$ after 6 ns. Chisholm and Janischewskyj [22] "fixed" this discrepancy introducing a fictitious grounding impedance (initially about $60 \Omega$ ) of the perfectly conducting plane. We will discuss further the interpretation of ground reflections observed in small-scale experiments in Section IV, and the uniform transmission line representation of a tall object on a conducting ground plane in Section V.

\section{B. 95-cm High-Conductor Simulating the CN Tower}

(Bermudez et al. [17])

Fig. 6 shows a perfectly conducting conical conductor having a short bulge (see Fig. 15(b) in Appendix) and representing the

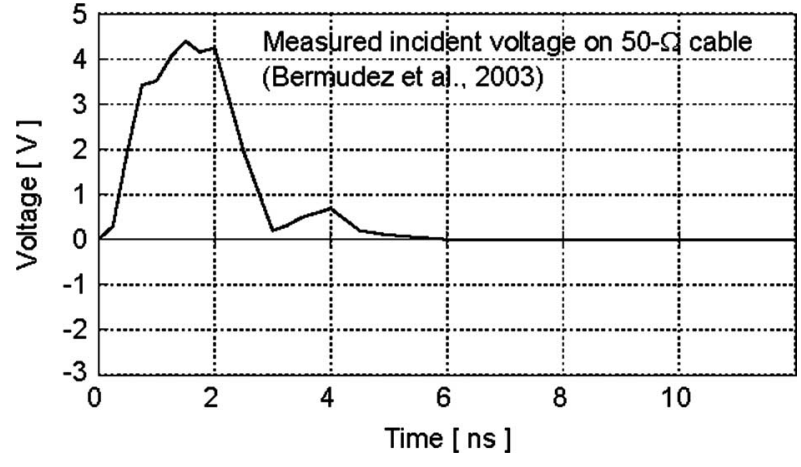

Fig. 7. Incident voltage waveform measured by Bermudez et al. [17] at the top end of the 2-m long 50- $\Omega$ cable. It was doubled in magnitude and used as the source voltage in the FDTD simulation. The magnitude doubling was needed to account for the voltage divider formed by the $50-\Omega$ series resistor and the characteristic impedance of the cable $(8.8 \mathrm{~V} \times 50 \Omega /(50 \Omega+50 \Omega)=$ $4.4 \mathrm{~V})$. Note that, in both the experiment of Bermudez et al. [17] and the FDTD simulation, the cables are long enough $(2 \mathrm{~m}$ in the experiment and $81 \mathrm{~cm}$ in the simulation) to separate the incident and reflected pulses in voltage records at the source.

553-m CN Tower in Toronto, Canada, which is placed between two horizontal conducting planes $94.5 \mathrm{~cm}$ apart. This configuration, to be analyzed using the FDTD method, simulates a small-scale experiment carried out by Bermudez et al. [17]. We use a $94.5-\mathrm{cm}$ conductor in the FDTD simulation instead of the 95-cm conductor employed by Bermudez et al. [17] because we represent it using rectangular cells having a vertical dimension of $1.5 \mathrm{~cm}$. A voltage pulse having an amplitude of $4.4 \mathrm{~V}$, shown in Fig. 7, is applied to the top of the $94.5-\mathrm{cm}$ tower through a $50-\Omega$ coaxial cable that is simulated in the same manner as the cable used in Section III-A. Voltage between the center conductor and the outer shield of this coaxial cable is monitored near the source ( $81 \mathrm{~cm}$ above the cone apex). The round-trip time of a traveling wave between the voltage measurement point and the lower end of the cable is $6 \mathrm{~ns}(=2 \times 81 \mathrm{~cm} / 27 \mathrm{~cm} / \mathrm{ns})$. Since the round-trip time is greater than the total pulse duration, about $5 \mathrm{~ns}$, the incident and reflected pulses should be well separated in voltage records at the source. Calculations were performed up to $12 \mathrm{~ns}$ with a time increment of $0.01 \mathrm{~ns}$. Note that in the experiment of Bermudez et al. [17], the voltage measurement point was $2 \mathrm{~m}$ (versus $81 \mathrm{~cm}$ in our simulation) above the cone apex. This is why no reflection is seen up to $12 \mathrm{~ns}$ in Fig. 7, but this does not influence the analysis presented here.

Fig. 8 shows the voltage waveform measured by Bermudez et al. [17] (thinner solid line) and that calculated using the FDTD method (thicker solid line) for the configuration shown in Fig. 6. The voltage waveform corresponding to the case of a $240-\Omega$ uniform transmission line, representing the cone, short-circuited at its bottom end, is also shown as a reference (broken line in Fig. 8). The latter waveform was calculated by using the Bergeron method (Dommel 1969 [24]). Note that the incident voltage pulse (see Fig. 7) is not shown in Fig. 8, only voltage pulses reflected from the top and bottom of the vertical conductor (tower model). In the FDTD simulation, the magnitude of the incident voltage pulse is $V_{\text {inc }}=4.4 \mathrm{~V}$ (see Fig. 7), and that of the first reflected voltage pulse from the junction between the coaxial cable $\left(Z_{\text {cable }}=50 \Omega\right)$ and the apex of 


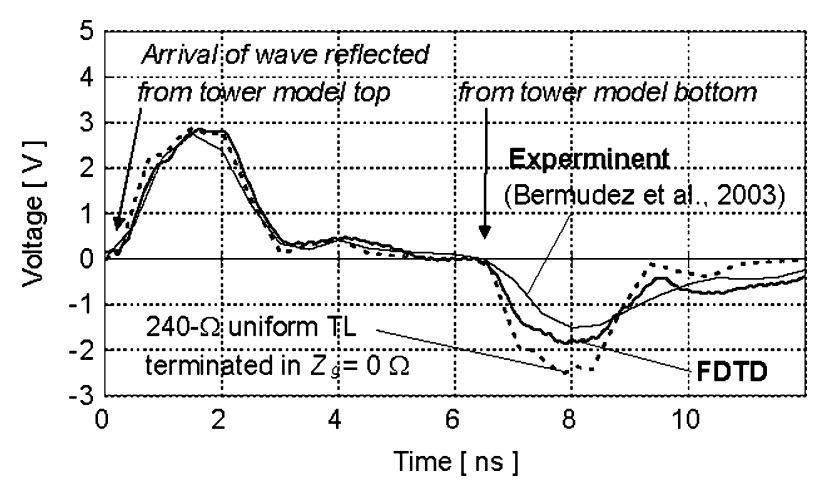

Fig. 8. Voltage waveforms at the top end of the 50- $\Omega$ cable (at the source) measured (thinner solid line) by Bermudez et al. [17] and calculated (thicker solid line) using the FDTD method for the configuration shown in Fig. 6. Also shown is the waveform (broken line) corresponding to a $240-\Omega$ uniform transmission line short-circuited at its bottom end, calculated using the Bergeron method (Dommel [24]). Note that the incident voltage pulse (see Fig. 7) is not shown here, only voltage pulses reflected from the top and bottom of the tower model.

the conical conductor ( $Z_{\text {cone }}$ is unknown) is $V_{\text {refl }}=2.85 \mathrm{~V}$ (see Fig. 8). From (2) with $V_{\text {inc }}=4.4 \mathrm{~V}, V_{\text {refl }}=2.85 \mathrm{~V}$, and $Z_{\text {cable }}=50 \Omega$, one can evaluate the characteristic impedance of the conical conductor to be $Z_{\text {cone }}=235 \Omega$. Also, from the measured magnitudes of the incident and reflected voltage pulses $\left(V_{\text {inc }}=4.4 \mathrm{~V}, V_{\text {refl }}=2.77 \mathrm{~V}\right.$; see Figs. 7 and 8$)$, one can evaluate the characteristic impedance of the conical conductor to be $Z_{\text {cone }}=220 \Omega$. The FDTD-estimated value of the characteristic impedance $235 \Omega$ is $7 \%$ higher than the experimental value $220 \Omega$ and about $10 \%$ lower than the theoretical value $260 \Omega$ calculated using (3). It is worth noting that the equation for characteristic impedance of a conical conductor excited at its apex, proposed by Sargent and Darveniza [15] yields $240 \Omega$ for the cone shown in Fig. 6. This equation is reproduced as follows:

$$
Z_{C}(\alpha)=60 \ln (\sqrt{2} / \sin \alpha)
$$

where $\alpha$ is the half-cone angle. The FDTD-estimated value 235 $\Omega$ is similar to the theoretical and experimental values.

As seen in Fig. 8, the FDTD-simulated waveform well reproduces the measured one. Bermudez et al. [17] have demonstrated that a model, which comprises a $50-\Omega$ uniform transmission line representing the cable and a $240-\Omega$ uniform transmission line representing the vertical conical conductor terminated at its bottom in a 60- $\Omega$ lumped grounding impedance, is capable of reproducing the magnitude of the second reflection (voltage wave reflected from the bottom-conducting plane) measured in their small-scale experiment (see the negative, thinner solid line pulse in Fig. 8). We will further discuss this latter model in Section V.

\section{INTERPRETATION OF GROUND REFLECTIONS ARRIVING AT THE TOWER TOP}

As noted in Section III, ground reflections observed at the top of small-scale tower models are smaller than expected for the case of perfect reflection (current reflection coefficient equal to 1) at the grounding plane and unattenuated upward propagation of reflected waves. This experimental result can be interpreted in three different ways: 1) The current reflection coefficient at the

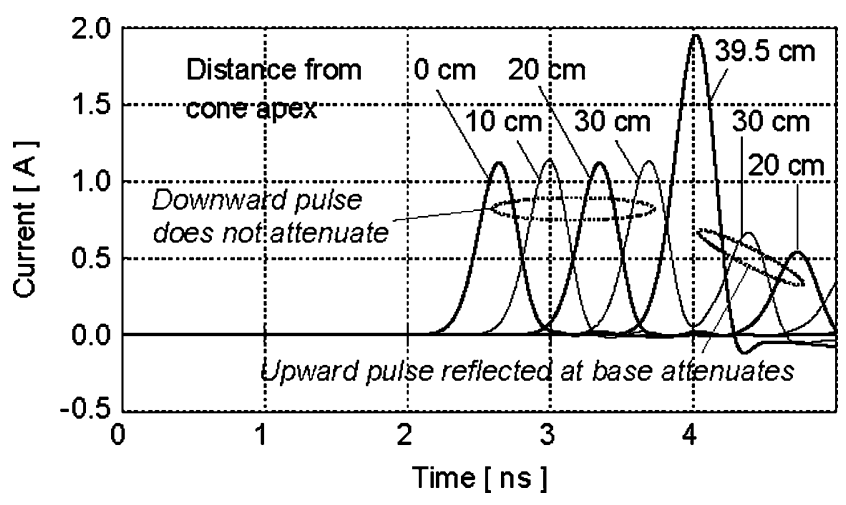

Fig. 9. Current waveforms, calculated using the FDTD method for the configuration shown in Fig. 4, at different vertical distances from the cone apex. In these calculations, we employed a voltage source that produced a Gaussian pulse having an amplitude of $200 \mathrm{~V}$ and a half-peak width of $0.33 \mathrm{~ns}$.

grounding plane is less than 1 and upward-propagating waves experience no attenuation. 2) The current reflection coefficient at the ground plane is equal to 1 , and the observed current deficit at the tower top is due to attenuation of upward propagating reflected waves. 3) The current reflection coefficient at the grounding plane is less than 1 , and upward-propagating reflected waves suffer attenuation. In the following, we will examine the current reflection coefficient at the grounding plane, using FDTD simulations and image theory. We will show that this coefficient should be close to 1 , which, along with the observed attenuation of the ground reflection, rules out interpretations 1) and 3).

Fig. 9 shows current waveforms, calculated using the FDTD method for the configuration shown Fig. 4, at different vertical distances along the cone axis from its apex. In these calculations, we employed a voltage source that produced a Gaussian pulse having an amplitude of $200 \mathrm{~V}$ and a half-peak width of $0.33 \mathrm{~ns}$. Before $4 \mathrm{~ns}$, the amplitude of current pulse, propagating downward along the surface of the conical conductor, away from its apex, is constant $(1.12 \mathrm{~A}$; see the four pulses at distances of 0 to $30 \mathrm{~cm}$ from the cone apex in Fig. 9). This is because a conical conductor (below a horizontal conducting plane) behaves as a uniform transmission line when a current pulse propagates away from the apex of the cone. The amplitude of current pulse, which reflects from the ground plane and propagates toward the cone apex (see the two pulses labeled $30 \mathrm{~cm}$ and $20 \mathrm{~cm}$ after $4 \mathrm{~ns}$ in Fig. 9), decreases with decreasing distance to the cone apex [see also Fig. 2(b)]. Thus, interpretation 1), adopted by Chisholm and Janischewskyj (1989 [22]) and Bermudez et al. [17], is ruled out.

Using the method of images, we can replace the configuration shown in Fig. 4 by its equivalent shown in Fig. 10, where the bottom perfectly conducting plane of Fig. 4 is replaced by the image-conducting cone. Current pulse reflected at the bottom plane in Fig. 4 corresponds to the upward-propagating current pulse in Fig. 10 when it passes through the zero-potential plane. This upward-propagating pulse injected into the upper conical conductor must be identical (due to symmetry) to the downward-propagating pulse injected into the lower conical conductor. In other words, there must be a perfect reflection (reflected current equal to incident current) at the perfectly 


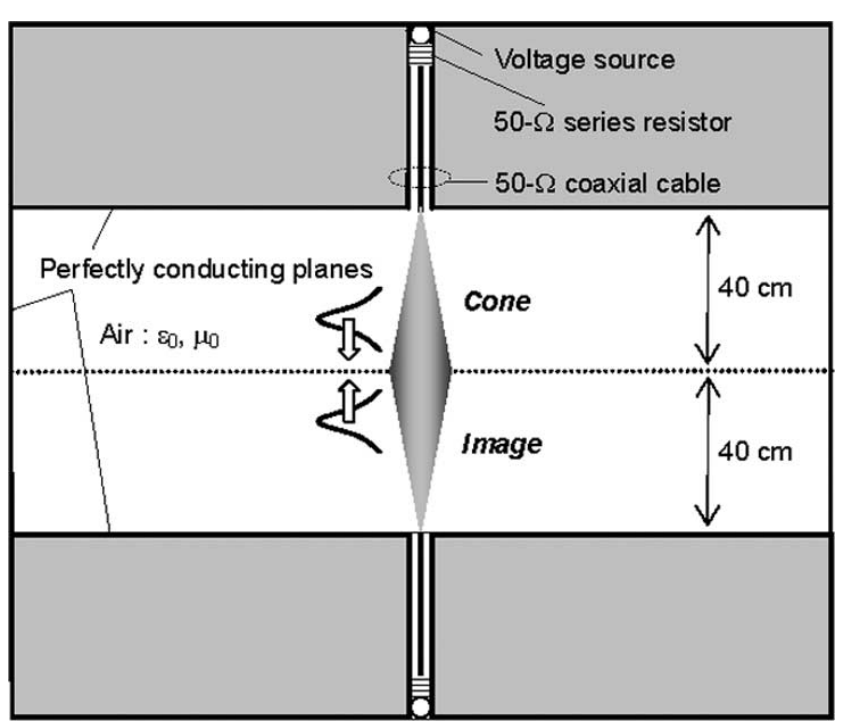

Fig. 10. Configuration equivalent to that shown in Fig. 4 for examining the production of the first reflection at the bottom of the cone, in which the bottom plane, assumed to be perfectly conducting, is replaced by the image cone. The position of that plane (imaginary zero-potential plane in this equivalent configuration) is indicated by horizontal dotted line.

conducting plane, that is, the current reflection coefficient must be equal to 1 . Therefore, a significant reduction in reflected current magnitude, relative to the incident current wave magnitude, at a perfectly conducting ground plane, or current reflection coefficient less than 1 , cannot occur unless there is a lumped impedance (grounding impedance) between the bottom of the conductor and the reference ground plane. This argues against both interpretations 1) and 3).

From the above results, it appears that the deficit of current observed at the tower model top is only due to attenuation of current waves propagating upward, toward the tower model top, as opposed to being due to nonperfect reflection at the grounding plane or both.

\section{TRANSMisSion Line RePRESENTATION OF A TALL ObJECT ON A GROUND PLANE}

In Sections II and IV, we have shown that a current pulse does not attenuate when it propagates from the apex of a conical conductor to its base but it does attenuate significantly when it propagates from the base of a conical conductor to its apex. Therefore, a transmission-line representation of the configuration shown in Fig. 4 should be a circuit, a portion of which has properties that are dependent on the direction of propagation. Such circuit is shown in Fig. 11(a). In this model, the conical conductor is represented by two lossless transmission lines, one of which is a uniform line for waves propagating away from the cone apex, and the other is a nonuniform line, whose characteristic impedance increases with decreasing distance to the cone apex, for waves propagating toward the cone apex. This representation reproduces (at least qualitatively) the decrease in magnitude of a current pulse propagating toward the cone apex observed in the FDTD simulations. However, such a model is not well suited for engineering applications, since it requires

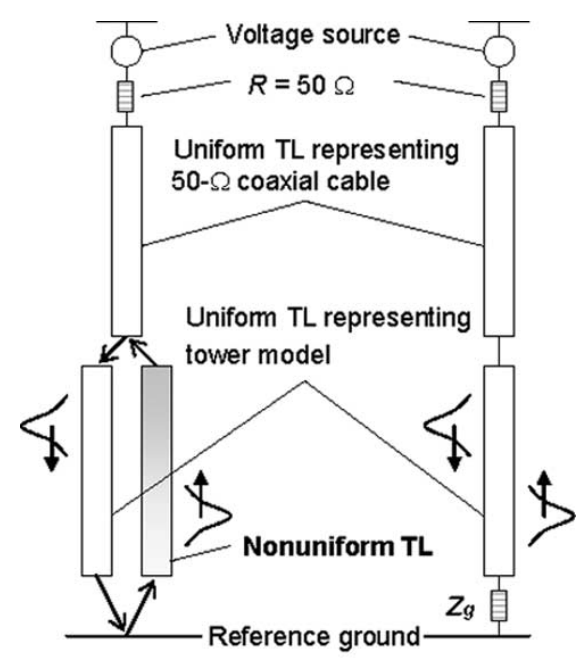

(a)

(b)

Fig. 11. Transmission line (TL) representations of the configurations shown in Figs. 4 and 6. (a) Conical conductor located between two horizontal conducting planes is represented by two lossless transmission lines, one of which is a uniform line for waves propagating away from the cone apex and the other is a nonuniform line, whose characteristic impedance increases with decreasing distance to the cone apex, for waves propagating toward the cone apex. (b) Conical conductor located between two horizontal conducting planes is represented by a single lossless, uniform transmission line (for both downward- and upward-propagating waves) terminated at its bottom end in a fictitious grounding impedance $Z_{g}$ of the bottom conducting plane. This representation was used by Bermudez et al. [17].

switching between the two transmission lines depending on the direction of propagation. Further, there is no formula to calculate the characteristic impedance of the nonuniform transmission line representing a conical conductor for a current pulse propagating toward the cone apex.

Fig. 11(b) shows an alternative representation of the configuration shown in Fig. 4, which is a single lossless uniform transmission line terminated in a fictitious lumped grounding impedance, $Z_{g}$, of the bottom conducting plane. This model was adopted by Bermudez et al. [17] who, in effect, lumped at the base of the cone the distributed current attenuation due to partial downward reflections in the nonuniform line, representing the cone for upward traveling waves in Fig. 11(a). Such an approach allowed them to eliminate the nonuniform line needed in the model shown in Fig. 11(a), but required a fictitious lumped resistor at the cone base.

Fig. 12 shows the voltage waveforms at the top of the $50-\Omega$ cable (at the voltage source), calculated using the model shown in Fig. 11(b), and that measured by Chisholm and Janischewskyj [22]. We assume the lumped grounding impedance to be $60 \Omega$ or equal to $60 h /(c t)$, where $h$ is the height of the cone, $c$ is the velocity of light, and $t$ is the time, in the traveling wave calculations using the Bergeron method (Dommel [24]). Note that $60 \mathrm{~h} /(\mathrm{ct})$, suggested by Chisholm and Janischewskyj [22], is the characteristic impedance of two horizontal conducting planes excited at the center by a cylindrically expanding TEM wave (Marcuvitz [25]). In the calculations employing the timevarying lumped grounding impedance, we assumed $t$ to start from $h / c$, following the method employed by Chisholm and Janischewskyj [22]. The waveform calculated assuming the 


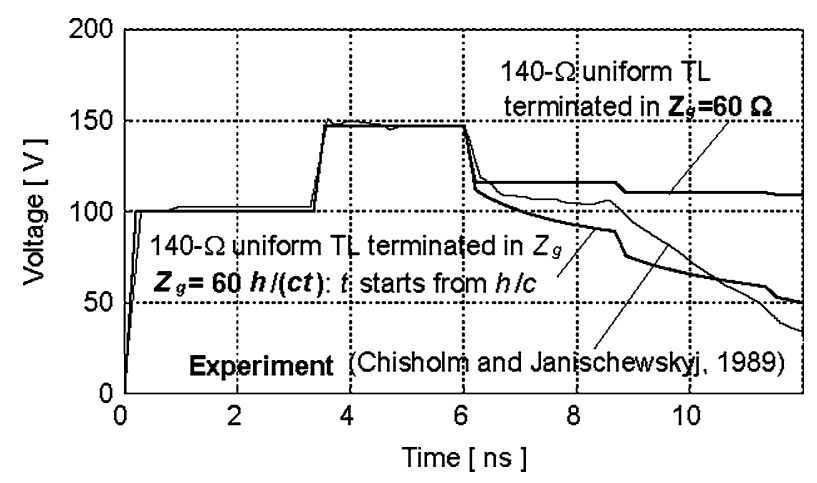

Fig. 12. Voltage waveforms at the top end of the 50- $\Omega$ cable (at the voltage source) measured by Chisholm and Janischewskyj [22] and calculated using the model shown in Fig. 11(b). We assume the lumped grounding impedance to be $60 \Omega$ or equal to $60 h /(c t)$, where $h$ is the height of the cone, $c$ is the velocity of light, and $t$ is the time, in traveling wave calculations using the Bergeron method (Dommel [24]). In the calculations employing the time-varying lumped grounding impedance, we assume $t$ to start from $h / c$, following the method employed by Chisholm and Janischewskyj [22].

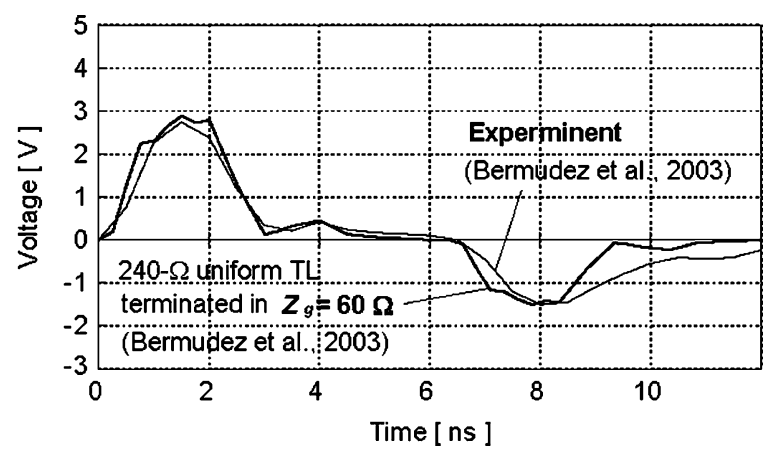

Fig. 13. Voltage waveforms at the top end of the $50-\Omega$ cable (at the voltage source) measured and calculated for the model shown in Fig. 11(b), both taken from Bermudez et al. [17]. In the calculations, the constant characteristic impedance of the $95-\mathrm{cm}$ conical tower was set to $240 \Omega$ and the lumped grounding impedance was assumed to be $60 \Omega$. Note that the incident voltage pulse (see Fig. 7) is not shown here, only voltage pulses reflected from the top and bottom of the tower model.

apparent grounding impedance to follow $60 \mathrm{~h} /(\mathrm{ct})$ reproduces the measured waveform quite well, while the uniform transmission line model with $Z_{g}=60 \Omega$ does not yield a good agreement with the experiment after $9 \mathrm{~ns}$. Note that all the representations discussed in this paragraph constitute an engineering approximation to account for the neglected attenuation of upward-propagating waves.

Fig. 13 shows voltage waveforms at the top of the $50-\Omega$ cable (at the voltage source) measured by Bermudez et al. [17] and that calculated by these researchers based on the model shown in Fig. 11(b). In the calculations, the constant characteristic impedance of the $95-\mathrm{cm}$ conical tower was set to $240 \Omega$ and the lumped grounding impedance was assumed to be $60 \Omega$ (Bermudez et al. [17]). As seen in Fig. 13, the uniform transmission line terminated in a constant $60-\Omega$ grounding impedance reasonably well reproduces the voltage waveform measured at the model tower top.

The engineering model shown in Fig. 11(b) is justified in calculating the voltage or current at the top of the cone at the time when the first reflection from the ground plane arrives.

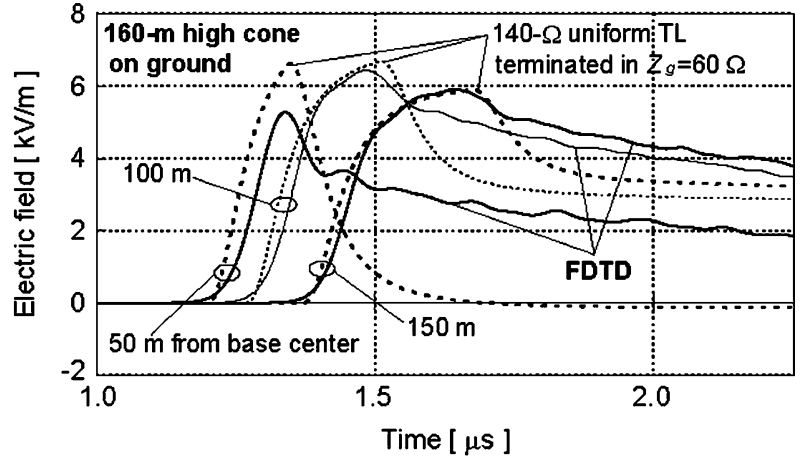

(a)

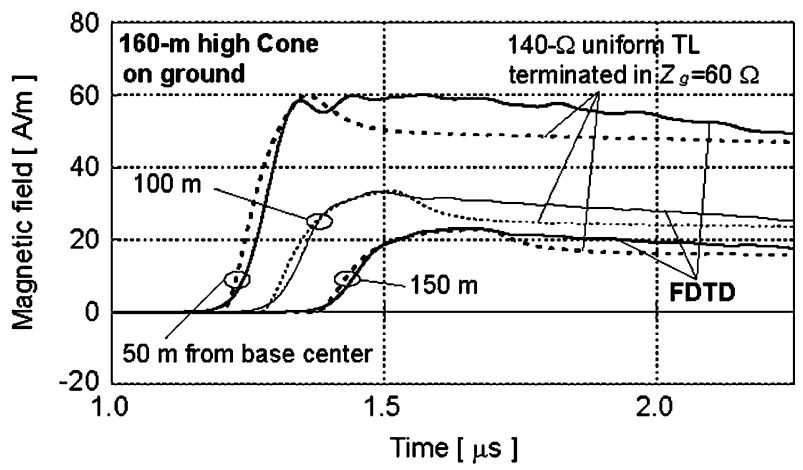

(b)

Fig. 14. (a) Vertical electric and (b) azimuthal magnetic field waveforms due to a lightning strike to a perfectly conducting cone, on a perfectly conducting plane at horizontal distances of 50,100, and $150 \mathrm{~m}$ from the base center of the cone. The cone has a height of $160 \mathrm{~m}$ and a base radius of $32 \mathrm{~m}$. Solid-line curves represent FDTD calculations in which excitation is applied between the cone apex and a second perfectly conducting plane in a configuration similar to that shown in Fig. 4. A current pulse representative of lightning subsequent return strokes (Nucci et al. [26]) was injected into the cone. Broken-line curves represent calculations based on the uniform transmission line model shown in Fig. 11(b). We set the characteristic impedance of the transmission line representing the cone to $Z_{\text {cone }}=140 \Omega$ and the lumped grounding impedance to $Z_{g}=60 \Omega$, so that the current reflection coefficient at the grounding plane was $\rho_{b}=0.4$.

However, current distribution along the conical conductor, particularly near its base, may be not adequately reproduced in the model assuming that the cone is a uniform transmission line (for both downward- and upward-propagating waves) that is terminated at its bottom end in a relatively high fictitious grounding impedance. Note that this latter representation predicts the same current waveform at all heights along the cone, while in reality the upward-moving waves are expected to suffer attenuation and dispersion [see Fig. 2(b)].

Fig. 14 shows vertical electric and azimuthal magnetic field waveforms due to a lightning strike to a perfectly conducting cone on a perfectly conducting ground plane at horizontal distances of 50,100, and $150 \mathrm{~m}$ from the base center of the cone. This cone has a height of $160 \mathrm{~m}$ and a base radius of $32 \mathrm{~m}$. The half-cone angle of this cone is $\alpha=11.3^{\circ}\left(=\tan ^{-1}(32 / 160)\right)$, equal to that of the cone shown in Fig. 4, and its characteristic impedance, according to (3), is equal to $140 \Omega$. Solid-line curves represent FDTD calculations in which excitation is applied between the cone apex and a second perfectly conducting plane in a configuration which is a $400: 1(160 \mathrm{~m}: 0.4 \mathrm{~m})$ version 
of that shown in Fig. 4. The use of the top conducting plane is equivalent to the assumption that the lightning channel can be represented by a perfectly conducting inverted cone whose half-cone angle is equal to that of the conical strike object. Such a configuration allows one to produce an unattenuated incident wave. We set output waveform of the voltage source so that a current pulse thought to be typical for lightning subsequent return strokes (Nucci et al. [26]) is injected into the apex of the cone. Note that fields on the bottom-conducting plane shown in Fig. 14 are not influenced by the reflection of waves from the top conducting plane. Broken-line curves in Fig. 14 represent calculations based on the uniform transmission line model shown in Fig. 11(b). We set the characteristic impedance of the $160-\mathrm{m}$ high cone to $Z_{\text {cone }}=140 \Omega$ and the lumped grounding impedance to $Z_{g}=60 \Omega$. Thus, the current reflection coefficient at the bottom conducting plane is $\rho_{\mathrm{b}}=0.4$. Note that Janischewskyj et al. [5], from their analysis of five current waveforms measured $474 \mathrm{~m}$ above ground on the $\mathrm{CN}$ tower, inferred $\rho_{\mathrm{b}}$ to vary from 0.34 to 0.43 , and Fuchs [27], from 13 simultaneous current measurements at the top and bottom of the Peissenberg tower, found $\rho_{\mathrm{b}}$ to vary from 0.64 to 0.81 .

It is clear from Fig. 14 that the azimuthal magnetic field waveforms calculated using the uniform transmission line model terminated in $Z_{g}=60 \Omega$ agree reasonably well with those calculated using the FDTD method. Also, magnitudes of the vertical electric field calculated using the uniform transmission line model agree reasonably well with those calculated using the FDTD method: The difference in peaks at $50 \mathrm{~m}$ is about $20 \%$ and decreases with increasing distance. On the other hand, at a distance of $50 \mathrm{~m}$, the vertical electric field predicted by the uniform transmission line model decays much faster than follows from the FDTD simulation and even exhibits a zero-crossing at $1.7 \mu \mathrm{s}$. For more distant observation points $(100$ and $150 \mathrm{~m})$, the uniform transmission line model yields more reasonably looking electric field waveshapes. The fast electric field decay at $50 \mathrm{~m}$ predicted by the uniform transmission line model is due to the fact that attenuation of the ground-reflected current wave (relative to the perfect-reflection case) is lumped at the cone base, while in reality (and in the FDTD simulation) current attenuation is distributed along the cone. The smaller the distance to the cone, the shorter the cone section "seen" at the observation point, and the more pronounced the difference between lumped attenuation and distributed attenuation of ground-reflected current waves.

\section{CONCLUSION}

In this paper, we have examined small-scale experiments conducted by Chisholm and Janischewskyj [22] and Bermudez et al. [17] to study the interaction of lightning with towers. In these experiments employing the TDR, the tower was represented by a conical conductor placed between two horizontal conducting planes.

Using the FDTD simulations, we have shown that the waveguiding properties of a biconical antenna depend on the direction of propagation: A current pulse suffers no attenuation when it propagates from the apex of the conical conductor to its base, but

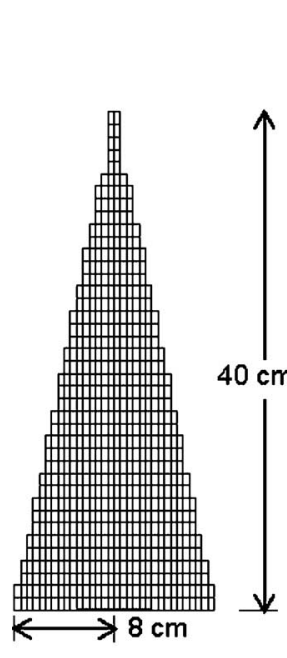

(a)

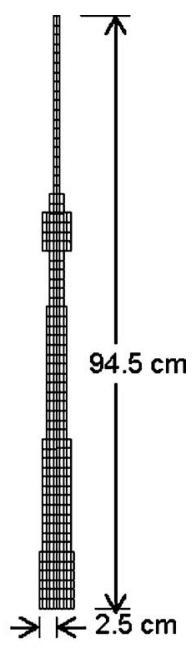

(b)
Fig. 15. Side views of conical conductors used in Sections III-A and B for the FDTD simulations presented in this paper. (a) A conical conductor of a height of $40 \mathrm{~cm}$ and base radius of $8 \mathrm{~cm}$, discretized using $0.5 \times 0.5 \times 1 \mathrm{~cm}^{3}$ rectangular cells. (b) A conical conductor of a height of $94.5 \mathrm{~cm}$ and base radius $2.5 \mathrm{~cm}$, having a short bulge at a height of $60 \mathrm{~cm}$, which is discretized using $0.5 \times 0.5 \times 1.5 \mathrm{~cm}^{3}$ rectangular cells. The conductors have staircase-circular cross sections, which are not shown here.

it attenuates significantly when it propagates from the base of the conical conductor to its apex. Thus, a conical conductor on a conducting plane cannot be viewed as a uniform transmission line for waves propagating upward, from the cone base to cone apex. Further, using the image theory, we show that the current reflection coefficient at the base of the conical conductor is close to 1 , so that the equivalent grounding impedance of the conducting plane is close to zero. Our analysis suggests that the relatively high grounding impedance (about $60 \Omega$, constant or decreasing with time) of conducting plane inferred by Chisholm and Janischewskyj [22] and Bermudez et al. [17] from their small-scale experiments is fictitious and should be viewed as an engineering approximation to account for the neglected attenuation of upward propagating waves. When the dependence of cone's waveguiding properties on the direction of propagation is taken into account, the results of the small-scale experiments simulating lightning strikes to towers can be interpreted without invoking the fictitious grounding impedance of conducting plane.

Representation of a vertical strike object by a uniform transmission line terminated in a fictitious grounding impedance is justified in computing the voltage or current at the top of the object at the time when the first reflection from the ground plane arrives. Further, this representation is apparently justified in computing lightning-generated magnetic fields and relatively distant electric fields. However, it may be inadequate for calculating electric fields at distances that are considerably smaller than the height of the object.

\section{APPENDIX}

\section{FDTD REPRESENTATION OF TOWER MODELS}

Fig. 15(a) and (b) shows the side views of conical conductors used in Sections III-A and B, respectively, discretized using 
rectangular cells for the FDTD simulations presented in this paper. The conductors have staircase-circular cross-sections, which are not shown here.

\section{ACKNOWLEDGMENT}

The authors would like to thank J. L. Bermudez, W. A. Chisholm, F. Heidler, C. A. Nucci, and F. Rachidi for discussions.

\section{REFERENCES}

[1] K. Berger, R. B. Anderson, and H. Kroninger, "Parameters of lightning flashes," Electra, vol. 80, pp. 223-237, 1975.

[2] B. N. Gorin, V. I. Levitov, and A. V. Shkilev, "Lightning strikes to the Ostankino tower," Elektrichestvo, no. 8, pp. 19-23, 1977. (in Russian).

[3] E. Montandon and B. Beyeler, "The lightning measuring equipment on the Swiss PTT telecommunication tower at St. Chrischona, Switzerland," in Proc. 22th Int. Conf. Lightning Protection, Budapest, Hungary, Sep. 1994, Paper R 1c-06.

[4] T. Zundl, "Lightning current and LEMP calculations compared to measurements gained at the Peissenberg tower," in Proc. 22th Int. Conf. Lightning Protection, Budapest, Hungary, Sep. 1994, Paper R 1c-08.

[5] W. Janischewskyj, V. Shostak, J. Barratt, A. M. Hussein, R. Rusan, and J.-S. Chang, "Collection and use of lightning return stroke parameters taking into account characteristics of the struck object," in Proc. 23rd Int. Conf. Lightning Protection, Florence, Italy, 1996, pp. 16-23.

[6] H. Goshima, H. Motoyama, A. Asakawa, A. Wada, T. Shindo, and S. Yokoyama, "Characteristics of electromagnetic fields due to lightning stroke current to a high stack in winter lightning," Trans. Inst. Electr. Eng. Jpn., vol. 120-B, no. 1, pp. 44-49, Jan. 2000.

[7] V. A. Rakov, "Transient response of a tall object to lightning," IEEE Trans. Electromagn. Compat., vol. 43, no. 4, pp. 654-661, Nov. 2001.

[8] F. Rachidi, V. A. Rakov, C. A. Nucci, and J. L. Bermudez, "Effect of vertically extended strike object on the distribution of current along the lightning channel," J. Geophys. Res., vol. 107, no. D23, Dec. 2002.

[9] J. L. Bermudez, M. Rubinstein, F. Rachidi, F. Heidler, and M. Paolone, "Determination of reflection coefficients at the top and bottom of elevated strike objects struck by lightning," J. Geophys. Res., vol. 108, no. D14, Jul. 2003.

[10] H. Motoyama, W. Janischewskyj, A. M. Hussein, R. Rusan, W. A. Chisholm, and J.-S. Chang, "Electromagnetic field radiation model for lightning strokes to tall structures," IEEE Trans. Power Del., vol. 11, no. 3, pp. 1624-1632, Jul. 1996.

[11] V. A. Rakov and M. A. Uman, "Review and evaluation of lightning return stroke models including some aspects of their application," IEEE Trans. Electromagn. Compat., vol. 40, no. 4, pp. 403-426, Nov. 1998.

[12] C. A. Jordan, "Lightning computation for transmission lines with overhead ground wires," General Electric Rev., vol. 37, pp. 130-137, 1934.

[13] C. F. Wagner and A. R. Hileman, "A new approach to the calculation of the lightning performance of transmission lines-Part III," AIEE Trans. Part III, vol. 79, pp. 589-603, 1960.

[14] Y. Baba and V. A. Rakov, "On the mechanism of attenuation of current waves propagating along a vertical perfectly conducting wire above ground: Application to lightning," IEEE Trans. Electromagn. Compat., vol. 47, no. 3, Aug. 2005.

[15] M. A. Sargent and M. Darveniza, "Tower surge impedance," IEEE Trans. Power App. Syst., vol. 88, no. 5, pp. 680-687, May 1969.

[16] W. A. Chisholm, Y. L. Chow, and K. D. Srivastava, "Lightning surge response of transmission towers," IEEE Trans. Power App. Syst., vol. 102, no. 9, pp. 3232-3242, Sep. 1983.

[17] J. L. Bermudez, F. Rachidi, W. A. Chisholm, M. Rubinstein, W. Janischewskyj, A. M. Hussein, V. Shostak, and J. S. Chang, "On the use of transmission line theory to represent a nonuniform vertically-extended object struck by lightning," in Proc. IEEE Symp. Electromagn. Compat., Boston, MA, Aug. 2003, pp. 501-504.

[18] C. A. Balanis, Antenna Theory: Analysis and Design, 2nd ed., New York: Wiley, 1997.
[19] K. S. Yee, "Numerical solution of initial boundary value problems involving Maxwell's equations in isotropic media," IEEE Trans. Antennas Propag., vol. 14, no. 3, pp. 302-307, Mar. 1966.

[20] A. Taflove and S. C. Hagness, Computational Electrodynamics: The Finite-Difference Time-Domain Method. Boston, MA: Artech House, Jun. 2000

[21] B. R. Kordi, R. Moini, and V. A. Rakov, "Comparison of lightning return stroke electric fields predicted by the transmission line and antenna theory models," in Proc. 15th Int. Zurich Symp. Electromagn. Compat., no. 102P2, Zurich, Switzerland, Feb. 2003, pp. 551-556.

[22] W. A. Chisholm and W. Janischewskyj, "Lightning surge response of ground electrodes," IEEE Trans. Power Del., vol. 4, no. 2, pp. 1329-1337, Apr. 1989.

[23] R. Thottappillil, J. Schoene, and M. A. Uman, "Return stroke transmission line model for stroke speed near and equal that of light," Geophys. Res. Lett., vol. 28, no. 18, pp. 3593-3596, Sep. 2001.

[24] H. W. Dommel, "Digital computer solution of electromagnetic transients in single and multiphase networks," IEEE Trans. Power App. Syst., vol. 88, no. 4, pp. 388-399, Apr. 1969.

[25] N. Marcuvitz, Waveguide Handbook, McGraw-Hill, New York, 1951, pp. 89-96.

[26] C. A. Nucci, G. Diendorfer, M. A. Uman, F. Rachidi, M. Ianoz, and C. Mazzetti, "Lightning return stroke current models with specified channel-base current: A review and comparison," J. Geophys. Res., vol. 95, no. D12, pp. 20 395-20 408, 1990.

[27] F. Fuchs, "On the transient behaviour of the telecommunication tower at the mountain Hoher Peissenberg," in Proc. 24th Int. Conf. Lightning Protection, vol. 1, Birminghan, U.K., 1998, pp. 36-41.

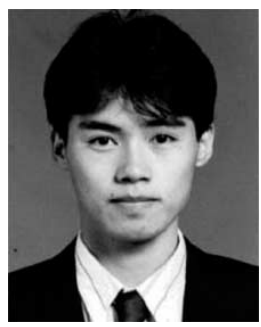

Yoshihiro Baba (S'95-M'99) received the B.S., M.S., and Dr.Eng. degrees from the University of Tokyo, Tokyo, Japan, in 1994, 1996, and 1999, respectively

$\mathrm{He}$ is a Associate Professor of the Department of Electrical Engineering, Doshisha University, Kyoto, Japan. From April 2003 to August 2004, he was a Visiting Scholar at the University of Florida, Gainesville, on sabbatical leave from Doshisha University. He is the author or coauthor of over 20 papers published in reviewed journals.

U.K.

Dr. Baba is a Member of AGU and Institution of Electrical Engineers (IEE),

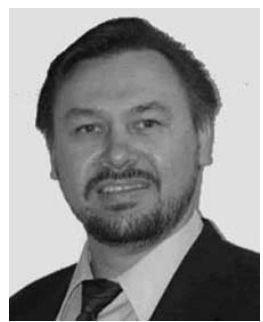

Vladimir A. Rakov (SM'96-F'03) received the M.S. and Ph.D. degrees from Tomsk Polytechnical University (Tomsk Polytechnic), Tomsk, Russia, in 1977 and 1983, respectively.

$\mathrm{He}$ is a Professor of the Department of Electrical and Computer Engineering, University of Florida (UF), Gainesville. He is the author or coauthor of one book, over 30 patents, and over 300 papers and technical reports on various aspects of lightning, with over 120 papers being published in reviewed journals. From 1977 to 1979, he worked as an Assistant Professor of Electrical Engineering at Tomsk Polytechnic. In 1978, he became involved in lightning research at the High Voltage Research Institute, a division of Tomsk Polytechnic, where from 1984 to 1994, he held the position of Director of the Lightning Research Laboratory. He is Chairman of the Technical Committee on Lightning of the biennial International Zurich Symposium on Electromagnetic Compatibility and former Chairman of the AGU Committee on Atmospheric and Space Electricity (CASE).

Dr. Rakov is a Fellow of AMS and a Member of AGU, ASEE, and SAE 